\title{
Existence of solutions for generalized mixed variational inequalities in reflexive Banach spaces
}

\author{
Zhong-Bao Wang*, Zi-Li Chen \\ Department of Mathematics, Southwest Jiaotong University, Chengdu, Sichuan 611756, P. R. China. \\ Communicated by S. S. Chang
}

\begin{abstract}
This paper is devoted to the solvability of generalized mixed variational inequalities in reflexive Banach spaces. We prove the existence of solutions of the generalized mixed variational inequalities for $f$ quasimonotone set-valued mappings without any assumption on bounded values. Furthermore, we give some conditions that guarantee the existence of solutions of the generalized mixed variational inequalities over unbounded closed convex subsets. Our results extend and improve some recent results from the literature. (C)2016 All rights reserved.
\end{abstract}

Keywords: Generalized mixed variational inequality, reflexive Banach space, existence, $f$-quasimonotonicity.

2010 MSC: 49J40, 47H10, 47H17.

\section{Introduction}

Throughout this paper, unless otherwise stated, we use $\rightarrow$ for convergence in strong sense and $\rightarrow$ for convergence in weak sense. Let $B$ be a reflexive Banach space with the dual space $B^{*}$, the norm and the dual pair between $B$ and $B^{*}$ be denoted by $\|\cdot\|$ and $\langle\cdot, \cdot\rangle$, respectively. Let $K$ be a nonempty convex subset of $B, R=(-\infty,+\infty), f: K \rightarrow R \cup\{+\infty\}$ be a proper, convex and lower semicontinuous function and $F: K \rightarrow 2^{B^{*}}$ be a set-valued mapping. We consider the following generalized mixed variational inequality (in short, GMVI): find $x \in K$ and $x^{*} \in F(x)$ such that

$$
\left\langle x^{*}, y-x\right\rangle+f(y)-f(x) \geq 0 \text { for all } y \in K .
$$

\footnotetext{
${ }^{*}$ Corresponding author

Email addresses: zhongbaowang@hotmail.com (Zhong-Bao Wang), zlchen@swjtu.edu.cn (Zi-Li Chen )
} 
This problem and its special cases has been studied by several authors and encountered in many applications, in particular, in mechanical problems and equilibrium problems. See [1, 2, 4, 5, 6, 7, 9, 14, 15, 20, 23, 24, [25, 28, 29] and the references therein.

If $F$ is the subdifferential of a finite-valued convex continuous function $\phi$ defined on $B$, then GMVI (1.1) reduces to the following nondifferential convex optimization problem:

$$
\min _{x \in K}\{f(x)+\phi(x)\} .
$$

If $f=0$ then GMVI (1.1) reduces to the following generalized variational inequality (in short, GVI): find $x \in K$ and $x^{*} \in F(x)$ such that

$$
\left\langle x^{*}, y-x\right\rangle \geq 0, \quad \text { for all } y \in K .
$$

If $f=0$ and $F$ is a single-valued mapping, then GMVI (1.1) reduces to the following classical variational inequality (in short, VI): find $x \in K$ such that

$$
\langle F(x), y-x\rangle \geq 0, \quad \text { for all } y \in K .
$$

Since 1960s, researchers have obtained many existence results of solutions for variational inequality problems; see [4, 6, 11, 13, 14, 16, 17, 19, 21, 22, 23, 24, 25, 27] and the references therein. Recently, By using the generalized projection operators in [2], Wong, Ansari and Yao[22] obtained existence results for solutions of the generalized variational inequality problem in a reflexive and smooth Banach space. By using the Lyapunov functional $G$ and the generalized $f$-projection operators in [23], Wu and Huang [25] established existence results for solutions of GMVI (1.1) in a reflexive and smooth Banach space. By using degree theory in [20], exceptional family of elements and the generalized $f$-projection operators, Wang and Huang [21] studied solvability results for the generalized set-valued variational inequality and its special cases in a reflexive, smooth and locally uniformly convex Banach space. Junlouchai and Plubtieng [14] presented the existence of solutions of GVI (1.3) involved the upper semicontinuous compact contractible valued multivalued mappings over compact convex subsets in a reflexive Banach space with a Fréchet differentiable norm. We note that if Banach spaces is only reflexive, existence results for solutions of the corresponding variational inequalities would not hold in [14, 21, 22, 25]. In addition, it is well known different kinds of generalized monotonicity of the operators play an important role in the theory of variational inequalities. By employing the notion of $\phi$-pseudomonotonicity of the operator, Zhong and Huang [28] studied the stability of Minty mixed variational inequality. By using the notion of the stable $\phi$-quasimonotonicity, Tang and Huang [19] studied the existence of solutions for the variational-hemivariational inequalities in reflexive Banach spaces. For more details, see [10, 19, 28, 29] and the references therein.

One may ask whether existence of solutions for GMVI (1.1) can be established or not under suitable monotonicity assumptions if Banach spaces are only reflexive.

The aim of this paper is to answer the above question. We would show that the existence of solutions of the generalized mixed variational inequalities for $f$-quasimonotone set-valued mappings, and give some conditions that guarantee the existence of solutions of the generalized mixed variational inequalities over unbounded closed convex subsets in reflexive Banach spaces. Moreover, the operator is not supposed to be bounded valued. The results presented in this paper extend and improve some corresponding results in [13, 14, 17, 21, 22, 25].

This rest of the paper is organized as follows. In Section 2, we introduce some basic notations and preliminary results. In Section 3 , we established some solvability results for the generalized mixed variational inequalities and its special cases.

\section{Preliminaries}

Definition 2.1. Let $f: K \rightarrow(-\infty,+\infty]$ be a function. 
(i) $f$ is said to be convex if, for all pairs $(x, y) \in K \times K$ and all $\lambda \in[0,1]$,

$$
f(\lambda x+(1-\lambda) y) \leq \lambda f(x)+(1-\lambda) f(y) ;
$$

(ii) $f$ is said to be lower semicontinuous if, for every $x \in K$,

$$
\liminf _{y \rightarrow x} f(y) \geq f(x) .
$$

Definition 2.2. Let $F: K \rightarrow 2^{B^{*}}$ be a set-valued mapping with nonempty values and $f: K \rightarrow(-\infty,+\infty]$ be a proper convex function.

(i) $F$ is said to be upper semicontinuous at $x_{0} \in K$ if, for any open set $V \subset B^{*}$ with $F(x) \subset V$, there exists an open neighborhood $U$ of $x_{0}$ such that $F(y) \subset V$ for all $y \in U \cap K$. If $F$ is upper semicontinuous at every $x \in K$, we say that $F$ is upper semicontinuous on $K$, where $B^{*}$ is equipped with the $w^{*}$-topology;

(ii) $F$ is said to be upper hemicontinuous on $K$ if the restriction of $F$ to every line segment of $K$ is upper semicontinuous with respect to the $w^{*}$-topology in $B^{*}$;

(iii) $F$ is said to be $f$-upper sign-continuous at $x \in K$ if the following implication holds

$$
\inf _{x_{t}^{*} \in F\left(x_{t}\right)}\left\langle x_{t}^{*}, x_{t}-x\right\rangle+f\left(x_{t}\right)-f(x) \geq 0 \Rightarrow \sup _{x^{*} \in F(x)}\left\langle x^{*}, y-x\right\rangle+f(y)-f(x) \geq 0 \quad \forall y \in K,
$$

where $x_{t}=x+t(y-x) \forall t \in(0,1)$. If $F$ is $f$-upper sign-continuous at every $x \in K$, we say that $F$ is $f$-upper sign-continuous on $K$.

\section{Remark 2.3.}

(i) If $f=0$, then the $f$-upper sign-continuity is precisely upper sign-continuity in [9].

(ii) If $F$ is upper hemicontinuous, then $F$ is $f$-upper sign-continuous. Indeed, if the assertion is not true, by definition of $f$-upper sign-continuity, we know there exists some $y \in K$, such that

$$
\left\langle x^{*}, y-x\right\rangle+f(y)-f(x)<0, \forall x^{*} \in F(x) .
$$

Since the set $\left\{\left\langle x^{*}, y-x\right\rangle+f(y)-f(x)<0\right\}$ is a $w^{*}$-open neighborhood of $F(x), K$ is convex and $F$ is upper hemicontinuous, setting $x_{t}=x+t(y-x) \in K$, for some $t \in(0,1)$ small enough, we have

$$
\left\langle x_{t}^{*}, y-x\right\rangle+f(y)-f(x)<0, \forall x_{t}^{*} \in F\left(x_{t}\right) .
$$

$x_{t}-x=t(y-x)$ implies that

$$
\left\langle x_{t}^{*}, x_{t}-x\right\rangle+t(f(y)-f(x))<0, \forall x_{t}^{*} \in F\left(x_{t}\right) .
$$

Since $f$ is convex, we know that

$$
f\left(x_{t}\right) \leq t f(y)+(1-t) f(x)
$$

and so $f\left(x_{t}\right)-f(x) \leq t(f(y)-f(x))$. Thus

$$
\left\langle x_{t}^{*}, x_{t}-x\right\rangle+f\left(x_{t}\right)-f(x)<0, \forall x_{t}^{*} \in F\left(x_{t}\right),
$$

which contradicts the assumption. So this assertion is true.

Definition 2.4. Let $F: K \rightarrow 2^{B^{*}}$ be a set-valued mapping with nonempty values and $f: K \rightarrow(-\infty,+\infty]$ be a proper function.

(i) $F$ is said to be monotone on $K$ if, for all $\left(x, x^{*}\right),\left(y, y^{*}\right)$ in the graph of $F$,

$$
\left\langle y^{*}-x^{*}, y-x\right\rangle \geq 0 ;
$$


(ii) $F$ is said to be pseudomonotone on $K$ if, for all $\left(x, x^{*}\right),\left(y, y^{*}\right)$ in the graph of $F$,

$$
\left\langle x^{*}, y-x\right\rangle \geq 0 \Rightarrow\left\langle y^{*}, y-x\right\rangle \geq 0
$$

(iii) $F$ is said to be $f$-pseudomonotone on $K$ if, for all $\left(x, x^{*}\right),\left(y, y^{*}\right)$ in the graph of $F$,

$$
\left\langle x^{*}, y-x\right\rangle+f(y)-f(x) \geq 0 \Rightarrow\left\langle y^{*}, y-x\right\rangle+f(y)-f(x) \geq 0 ;
$$

(iv) $F$ is said to be quasimonotone on $K$ if, for all $\left(x, x^{*}\right),\left(y, y^{*}\right)$ in the graph of $F$,

$$
\left\langle x^{*}, y-x\right\rangle>0 \Rightarrow\left\langle y^{*}, y-x\right\rangle \geq 0
$$

(v) $F$ is said to be $f$-quasimonotone on $K$ if, for all $\left(x, x^{*}\right),\left(y, y^{*}\right)$ in the graph of $F$,

$$
\left\langle x^{*}, y-x\right\rangle+f(y)-f(x)>0 \Rightarrow\left\langle y^{*}, y-x\right\rangle+f(y)-f(x) \geq 0 ;
$$

Remark 2.5.

(i) If $f=0$, then the $f$-pseudomonotonicity and $f$-quasimonotonicity of mappings are precisely pseudomonotonicity and quasimonotonicity, respectively.

(ii) We would like to point out that the $f$-pseudomonotonicity of mappings was used to study the $F$ complementarity problems and mixed variational inequalities in Banach spaces in [28, 29]. Especially, by using some examples, Zhong and Huang [28] showed that a $f$-pseudomonotone mapping may not be pseudomonotone, a pseudomonotone mapping may not be $f$-pseudomonotone and a $f$-pseudomonotone mapping may not be monotone in general.

(iii) It is clear that the following implications hold:

$$
\begin{aligned}
& \text { monotonicity } \Rightarrow \text { pseudomonotonicity } \Rightarrow \text { quasimonotonicity; } \\
& \text { monotonicity } \Rightarrow f \text {-pseudomonotonicity } \Rightarrow f \text {-quasimonotonicity. }
\end{aligned}
$$

By using some examples, Tang and Huang [19] showed that the inverse direction of each implication relationship mentioned above is false in general.

Lemma 2.6 ([8] $)$. Let $\tilde{K}$ be a nonempty subset of a Hausdorff topological vector space $E$ and $\bar{G}: \tilde{K} \rightarrow E$ be a set-valued mapping from $\tilde{K}$ into $E$ satisfying the following properties:

(i) $\bar{G}$ is a KKM mapping, i.e., for every finite subset $A$ of $\tilde{K}, \operatorname{conv} A \subset \bigcup_{x \in A} \bar{G}(x)$;

(ii) $\bar{G}(x)$ is closed in $E$ for every $x \in \tilde{K}$;

(iii) $\bar{G}\left(\bar{x}_{0}\right)$ is compact in $E$ for some $\bar{x}_{0} \in \tilde{K}$.

Then $\bigcap_{x \in \tilde{K}} \bar{G}(x) \neq \emptyset$.

Lemma 2.7 ([18]). Let $A_{1}$ be a nonempty convex set in a vector space and let $A_{2}$ be a nonempty compact convex set in a Hausdorff topological vector space. Suppose that $g$ is a real-valued function on $A_{1} \times A_{2}$ such that, for each fixed $a \in A_{1}, g(a, \cdot)$ is lower semicontinuous and convex on $A_{2}$, and for each fixed $b \in A_{2}$, $g(\cdot, b)$ is concave on $A_{1}$. Then

$$
\inf _{b \in A_{2}} \sup _{a \in A_{1}} g(a, b)=\sup _{a \in A_{1}} \inf _{b \in A_{2}} g(a, b) .
$$

\section{Main results}

It is known that a solution of the Minty mixed variational inequality is also a solution of the generalized mixed variational inequality, provided that $F$ is upper hemicontinuous with convex, $w *$-compact values, see Lemma 2.4 in [29]. Using essentially the similar argument, we show that the same is true under weaker assumptions. 
Lemma 3.1. Let $K$ be a nonempty convex subset of $B, F: K \rightarrow 2^{B^{*}}$ be an operator and $f: K \rightarrow R \cup\{+\infty\}$ be a proper and convex function. Suppose that, for every $x \in K$, there exists a convex neighborhood $V_{x}$ of $x$ and a $f$-upper sign-continuous operator $S_{x}: V_{x} \cap K \rightarrow 2^{B^{*}}$ with nonempty, $w^{*}$-compact and convex values satisfying $S_{x}(y) \subseteq F(y) \forall y \in V_{x} \cap K$. Moreover, if there exist $x \in K$ and a convex neighborhood $U$ of $x$ such that for all $y \in K \cap U$

$$
\left\langle y^{*}, x-y\right\rangle+f(x)-f(y) \leq 0 \quad \forall y^{*} \in F(y),
$$

then $x \in K$ is a solution of GMVI (1.1).

Proof. We claim that for each $y \in K \cap U \cap V_{x}$, there exists $x^{*} \in S_{x}(x)$ such that

$$
\left\langle x^{*}, y-x\right\rangle+f(y)-f(x) \geq 0 .
$$

For each $y \in K \cap U \cap V_{x}$, since $K \cap U \cap V_{x}$ is convex, $x_{t}=x+t(y-x) \in K \cap U \cap V_{x} \subset K \cap U \forall t \in(0,1)$. By (3.1), we have

$$
\left\langle x_{t}^{*}, x_{t}-x\right\rangle+f\left(x_{t}\right)-f(x) \geq 0, \forall x_{t}^{*} \in S_{x}\left(x_{t}\right)
$$

and so

$$
\inf _{x_{t}^{*} \in S_{x}\left(x_{t}\right)}\left\langle x_{t}^{*}, x_{t}-x\right\rangle+f\left(x_{t}\right)-f(x) \geq 0 .
$$

From $f$-upper sign-continuity of $S_{x}$, it follows that

$$
\sup _{x^{*} \in S_{x}(x)}\left\langle x^{*}, y-x\right\rangle+f(y)-f(x) \geq 0 .
$$

Since $S_{x}$ is $w *$-compact, there exists $x^{*} \in S_{x}(x) \subset F(x)$ such that

$$
\left\langle x^{*}, y-x\right\rangle+f(y)-f(x) \geq 0 \forall y \in K \cap U \cap V_{x} .
$$

So this assertion is true.

From $(3.2)$, it follows that

$$
\sup _{y \in K \cap U \cap V_{x}} \inf _{x^{*} \in S_{x}(x)}\left\langle x^{*}, x-y\right\rangle+f(x)-f(y) \leq 0 .
$$

As $S_{x}$ has $w^{*}$-compact and convex values and $f$ is convex, Lemma 2.7 implies

$$
\sup _{y \in K \cap U \cap V_{x}} \inf _{x^{*} \in S_{x}(x)}\left\langle x^{*}, x-y\right\rangle+f(x)-f(y)=\inf _{x^{*} \in S_{x}(x)} \sup _{y \in K \cap U \cap V_{x}}\left\langle x^{*}, x-y\right\rangle+f(x)-f(y)
$$

and so there exists some $x^{*} \in S_{x}(x)$ such that

$$
\left\langle x^{*}, y-x\right\rangle+f(y)-f(x) \geq 0, \forall y \in K \cap U \cap V_{x} .
$$

For any given $y \in K$, there exists $t^{\prime} \in(0,1)$ such that $x+t^{\prime}(y-x) \in K \cap U \cap V_{x}$. Equation (3.3) implies that

$$
\left\langle x^{*}, x+t^{\prime}(y-x)-x\right\rangle+f\left(x+t^{\prime}(y-x)\right)-f(x) \geq 0 .
$$

Since $f$ is convex, we know

$$
f\left(x+t^{\prime}(y-x)\right)-f(x) \leq t^{\prime} f(y)+\left(1-t^{\prime}\right) f(x)-f(x)=t^{\prime}(f(y)-f(x)) .
$$

Thus we get

$$
\left\langle x^{*}, y-x\right\rangle+f(y)-f(x) \geq 0 .
$$

Thus $x$ is a solution of GMVI 1.1 . The proof is complete. 
As the authors 3 pointed out if $F$ itself is $f$-upper sign-continuous and has nonempty, convex and $w^{*}$-compact values, then we can take in the lemma $V_{x}=K, S_{x}=F$. However, the lemma in its present form permits application to operators whose values are unbounded, such as cone-valued operators. If $f=0$, then Lemma 3.1 reduces to Lemma 2.1 of [3].

Theorem 3.2. Let $K$ be a nonempty bounded and closed convex subset of $B$ and $f: K \rightarrow R \cup\{+\infty\}$ be a proper and convex lower semicontinuous function. Suppose that $F: K \rightarrow 2^{B^{*}}$ is $f$-quasimonotone, and for every $x \in K$, there exists a convex neighborhood $V_{x}$ of $x$ and a $f$-upper sign-continuous operator $S_{x}: V_{x} \cap K \rightarrow 2^{B^{*}}$ with nonempty, $w^{*}$-compact and convex values satisfying $S_{x}(y) \subseteq F(y) \forall y \in V_{x} \cap K$. Then GMVI (1.1) has a solution.

Proof. Since $K$ be a nonempty bounded and closed convex subset of the reflexive Banach space $B, K$ is a $w^{*}$-compact set. Let $G: K \rightarrow 2^{K}$ be a set-valued mapping defined by

$$
G(y)=\left\{\hat{x} \in K,\left\langle y^{*}, \hat{x}-y\right\rangle+f(\hat{x})-f(y) \leq 0 \quad \forall y^{*} \in F(y)\right\} \forall y \in K .
$$

Obviously $G(y) \neq \emptyset \forall y \in K$. We prove that $G(y)$ is a closed subset of $K$. In fact, for any $x_{n} \in G(y)$ with $x_{n} \rightarrow \bar{x}$, we have

$$
\left\langle y^{*}, x_{n}-y\right\rangle+f\left(x_{n}\right)-f(y) \leq 0 \quad \forall y^{*} \in F(y) .
$$

The lower semicontinuity of $f$ implies that

$$
\left\langle y^{*}, \bar{x}-y\right\rangle+f(\bar{x})-f(y) \leq \liminf _{n \rightarrow+\infty}\left\langle y^{*}, x_{n}-y\right\rangle+\liminf _{n \rightarrow+\infty} f\left(x_{n}\right)-f(y) \leq 0 \quad \forall y^{*} \in F(y) .
$$

This shows that $G(y) \subset K$ is closed and so $G(y)$ is $w^{*}$-compact.

Next we consider two cases regarding $G$ : (i) $G$ is not a KKM mapping, and (ii) $G$ is a KKM mapping.

Case (i) If $G$ is not a KKM mapping, then, according to the definition of KKM mapping, there exist $t_{1}, t_{2}, \cdots, t_{n} \in[0,1], y_{1}, y_{2}, \cdots, y_{n} \in K, \sum_{i=1}^{n} t_{i}=1$ and $\bar{y}=\sum_{i=1}^{n} t_{i} y_{i}$ such that $\bar{y} \notin \bigcup G\left(y_{i}\right), i=1,2, \cdots, n$.

Then there exists $y_{i}^{*} \in F\left(y_{i}\right)$ such that

$$
\left\langle y_{i}^{*}, \bar{y}-y_{i}\right\rangle+f(\bar{y})-f\left(y_{i}\right)>0, \quad i=1,2, \cdots, n .
$$

Since $\left\langle y_{i}^{*}, \cdot-y_{i}\right\rangle+f(\cdot)-f\left(y_{i}\right)$ is lower semicontinuous at $\bar{y}$ in the Banach space $B$, there exists a convex neighborhood $U_{1}$ of $\bar{y}$ satisfying, for any $y \in K \cap U_{1}$, one has

$$
\left\langle y_{i}^{*}, y-y_{i}\right\rangle+f(y)-f\left(y_{i}\right)>0, \quad i=1,2, \cdots, n .
$$

$f$-quasimonotonicity of $F$ implies that for all $y^{*} \in F(y)$

$$
\left\langle y^{*}, y-y_{i}\right\rangle+f(y)-f\left(y_{i}\right) \geq 0, \quad i=1,2, \cdots, n .
$$

Since $\bar{y}=\sum_{i=1}^{n} t_{i} y_{i}$ and $f$ is convex, we have

$$
\left\langle y^{*}, y-\bar{y}\right\rangle+f(y)-f(\bar{y}) \geq\left\langle y^{*}, \sum_{i=1}^{n} t_{i} y-\sum_{i=1}^{n} t_{i} y_{i}\right\rangle+\sum_{i=1}^{n} t_{i} f(y)-\sum_{i=1}^{n} t_{i} f\left(y_{i}\right) \geq 0,
$$

and so

$$
\left\langle y^{*}, \bar{y}-y\right\rangle+f(\bar{y})-f(y) \leq 0 \quad \forall y \in K \cap U_{1} \forall y^{*} \in F(y) .
$$

By Lemma 3.1, we know $\bar{y}$ is a solution of GMVI 1.1).

Case (ii) If $G$ is a KKM mapping, then Lemma 2.6 implies that $x \in \bigcap_{y \in K} G(y) \neq \emptyset$. Thus we have for all $y \in K$

$$
\left\langle y^{*}, x-y\right\rangle+f(x)-f(y) \leq 0 \quad \forall y^{*} \in F(y) .
$$

By Lemma 3.1. we know $x$ is a solution of GMVI (1.1). The proof is complete. 
By taking $f=0$ in Theorem 3.2, we get the following corollary.

Corollary 3.3. Let $K$ be a nonempty bounded and closed convex subset of B. Suppose that $F: K \rightarrow 2^{B^{*}}$ is quasimonotone, and for every $x \in K$, there exists a convex neighborhood $V_{x}$ of $x$ and a upper sign-continuous operator $S_{x}: V_{x} \cap K \rightarrow 2^{B^{*}}$ with nonempty, $w^{*}$-compact and convex values satisfying $S_{x}(y) \subseteq F(y) \forall y \in$ $V_{x} \cap K$. Then GVI (1.3) has a solution.

Remark 3.4. Although we assume that $K$ is a bounded and closed set in reflexive Banach space $B$, we don't suppose that $K$ is nonempty convex and weakly compact in Proposition 2.1 of [3]. In addition, Corollary 3.3 extends and improves Theorem 3.1 in 14 in the following aspects:

(i) the Banach space $B$ may not has a Fréchet differentiable norm;

(ii) $K$ and $F(K)=\cup_{x \in K} F(x)$ may not be compact in $B^{*}$;

(iii) the upper semicontinuity of the mappings is relaxed to the upper sign-continuity.

Relaxing the constraint set $K$ of variational inequalities to the unbounded case, various coercivity conditions have been used [11, 13, 14, 16, 17, 19, 21, 22. In order to show the existence of solutions for GMVI (1.1) in unbounded constraint set, we give the following coercivity conditions:

$\left(C_{1}\right)$ Given $\hat{x} \in B$ and for any $\left\{x_{n}\right\} \subset K$ with $\left\|x_{n}\right\| \rightarrow+\infty$ as $n \rightarrow+\infty$, and any $\left\{u_{n}\right\}$ with $u_{n} \in F\left(x_{n}\right)$, there exist a positive integer $N$ and $\bar{x} \in K$ such that $\|\bar{x}-\hat{x}\| \leq\left\|x_{N}-\hat{x}\right\|$ and

$$
\left\langle u_{N}, \bar{x}-x_{N}\right\rangle+f(\bar{x})-f\left(x_{N}\right)<0 ;
$$

$\left(C_{2}\right)$ Given $\hat{x} \in B$ and for any $\left\{x_{n}\right\} \subset K$ with $\left\|x_{n}\right\| \rightarrow+\infty$ as $n \rightarrow+\infty$, and any $\left\{u_{n}\right\}$ with $u_{n} \in F\left(x_{n}\right)$, there exist a positive integer $N$ and $\bar{x} \in K$ such that $\|\bar{x}-\hat{x}\|<\left\|x_{N}-\hat{x}\right\|$ and

$$
\left\langle u_{N}, \bar{x}-x_{N}\right\rangle+f(\bar{x})-f\left(x_{N}\right) \leq 0 ;
$$

$\left(C_{3}\right)$ Given $\hat{x} \in B$, there exists a constant $\rho>0$ such that, for any $x \in K$ with $\|x-\hat{x}\|>\rho$, there exists $\bar{x} \in K$ satisfying $\|\bar{x}-\hat{x}\| \leq\|x-\hat{x}\|$ and $\sup _{u \in F(x)}\langle u, \bar{x}-x\rangle+f(\bar{x})-f(x)<0 ;$

$\left(C_{4}\right)$ Given $\hat{x} \in B$, there exists a constant $\rho>0$ such that, for any $x \in K$ with $\|x-\hat{x}\|>\rho$, there exists $\bar{x} \in K$ satisfying $\|\bar{x}-\hat{x}\|<\|x-\hat{x}\|$ and $\sup _{u \in F(x)}\langle u, \bar{x}-x\rangle+f(\bar{x})-f(x) \leq 0 ;$

$\left(C_{5}\right)$ Suppose that there exists a vector $\tilde{x} \in K$ such that the set

$$
L_{<}(\tilde{x}):=\left\{x \in K: \inf _{u \in F(x)}\langle u, x-\tilde{x}\rangle+f(x)-f(\tilde{x})<0\right\}
$$

is bounded (possibly empty).

Remark 3.5.

(i) If $f=0, B=R^{n}$ is a finite dimensional space, the conditions $\left(C_{1}\right)-\left(C_{4}\right)$ become the conditions $\left(C_{1}\right)-\left(C_{4}\right)$, respectively in [26]. If $f=0, B=H$ is a Hilbert space and $F$ is a single-valued mapping from $H$ to $H$, then the condition $\left(C_{5}\right)$ becomes $F$ satisfying the (HP) condition according to Definition 6.4 in [12. If $\hat{x}=0$ and $f=0$, the condition $\left(C_{4}\right)$ becomes the condition (3) of Theorem 2.1 in [3].

(ii) We firstly claim $\left(C_{4}\right) \Rightarrow\left(C_{2}\right)$. Indeed for any $\left\{x_{n}\right\} \subset K$ with $\left\|x_{n}\right\| \rightarrow+\infty$ as $n \rightarrow+\infty$, and any $\left\{u_{n}\right\}$ with $u_{n} \in F\left(x_{n}\right)$, since $\rho$ is a constant, there exists a positive integer $N$ such that $\left\|x_{N}-\hat{x}\right\|>\rho$. By $\left(C_{4}\right)$, there exists $\bar{x} \in K$ such that $\|\bar{x}-\hat{x}\|<\left\|x_{N}-\hat{x}\right\|$ and

$$
\left\langle u_{N}, \bar{x}-x_{N}\right\rangle+f(\bar{x})-f\left(x_{N}\right) \leq 0 .
$$

Hence the condition $\left(C_{2}\right)$ holds. 
Next, we prove that $\left(C_{3}\right) \Rightarrow\left(C_{1}\right)$. In fact, for any $\left\{x_{n}\right\} \subset K$ with $\left\|x_{n}\right\| \rightarrow+\infty$ as $n \rightarrow+\infty$, and any $\left\{u_{n}\right\}$ with $u_{n} \in F\left(x_{n}\right)$, since $\rho$ is a constant, there exists a positive integer $N$ such that $\left\|x_{N}-\hat{x}\right\|>\rho$. By $\left(C_{3}\right)$, there exist a positive integer $N$ and $\bar{x} \in K$ such that $\|\bar{x}-\hat{x}\| \leq\left\|x_{N}-\hat{x}\right\|$ and

$$
\left\langle u_{N}, \bar{x}-x_{N}\right\rangle+f(\bar{x})-f\left(x_{N}\right)<0 .
$$

Hence the condition $\left(C_{1}\right)$ holds.

(iii) Obviously $\left(C_{5}\right) \Rightarrow\left(C_{4}\right)$. Indeed, if the condition $\left(C_{5}\right)$ holds, then there exists $\tilde{x} \in K$ such that $L_{<}(\tilde{x})$ is bounded or empty. In this case, there exists $\rho_{3}>0$ such that $L_{<}(\tilde{x}) \subset B\left(\tilde{x}, \rho_{3}\right)$, where

$$
B\left(\tilde{x}, \rho_{3}\right)=\left\{x \in K:\|x-\hat{x}\| \leq \rho_{3}\right\} .
$$

Given $\hat{x} \in K$, we take $\rho=\max \left\{\rho_{3},\|\tilde{x}-\hat{x}\|\right\}$. If $x \in K$ is an arbitrary element such that $\|x-\hat{x}\|>\rho$, then we have $x \notin L_{<}(\tilde{x})$, that is,

$$
\inf _{u \in F(x)}\langle u, x-\tilde{x}\rangle+f(x)-f(\tilde{x}) \geq 0
$$

and so

$$
\sup _{u \in F(x)}\langle u, \tilde{x}-x\rangle+f(\tilde{x})-f(x) \leq 0 .
$$

For any $x \in K$ such that $\|x-\hat{x}\|>\rho$, taking $\bar{x}=\tilde{x}$, we know that the condition $\left(C_{4}\right)$ holds.

Theorem 3.6. Let $K$ be a nonempty and closed convex subset of $B$ and $f: K \rightarrow R \cup\{+\infty\}$ be a proper and convex lower semicontinuous function. Suppose that $F: K \rightarrow 2^{B^{*}}$ is $f$-quasimonotone, and for every $x \in K$, there exists a convex neighborhood $V_{x}$ of $x$ and a $f$-upper sign-continuous operator $S_{x}: V_{x} \cap K \rightarrow 2^{B^{*}}$ with nonempty, $w^{*}$-compact and convex values satisfying $S_{x}(y) \subseteq F(y) \forall y \in V_{x} \cap K$. If the condition $\left(C_{1}\right)$ holds, then GMVI (1.1) has a solution.

Proof. Let $K_{n}=\{x \in K:\|x-\hat{x}\| \leq n\},(n=1,2, \cdots)$. We note that $K_{n}$ is a nonempty bounded and closed convex subset of $B$. Without loss of generality, we assume that it starts from some $n$. By Theorem 3.2 , there exist $x_{n} \in K_{n}$ and $u_{n} \in F\left(x_{n}\right)$ such that

$$
\left\langle u_{n}, y-x_{n}\right\rangle+f(y)-f\left(x_{n}\right) \geq 0 \forall y \in K_{n} .
$$

If the sequence $\left\{x_{n}\right\}$ is unbounded, and without loss of generality, we assume that $\left\|x_{n}\right\| \rightarrow+\infty$. The condition $\left(C_{1}\right)$ implies that there exist a positive integer $N$ and $\bar{x} \in K$ such that $\|\bar{x}-\hat{x}\| \leq\left\|x_{N}-\hat{x}\right\| \leq N$ (and hence $\bar{x} \in K_{N}$ ) and

$$
\left\langle u_{N}, \bar{x}-x_{N}\right\rangle+f(\bar{x})-f\left(x_{N}\right)<0 \forall u_{N} \in F\left(x_{N}\right) .
$$

This contradicts with (3.5). Hence $\left\{x_{n}\right\}$ is bounded and so there exist a positive integer $M$ such that $\left\|x_{n}-\hat{x}\right\| \leq M$. Let $n=M+1$ in (3.5). For each $y \in K$, we can choose $t \in(0,1)$ such that $z=$ $x_{n}+t\left(y-x_{n}\right) \in K_{n}$. Setting $y=z$ in (3.5), we have

$$
\left\langle u_{n}, x_{n}+t\left(y-x_{n}\right)-x_{n}\right\rangle+f\left(x_{n}+t\left(y-x_{n}\right)\right)-f\left(x_{n}\right) \geq 0 .
$$

Since $f$ is convex, we know that

$$
f\left(x_{n}+t\left(y-x_{n}\right)\right) \leq t f(y)+(1-t) f\left(x_{n}\right)
$$

and so $f\left(x_{n}+t\left(y-x_{n}\right)\right)-f\left(x_{n}\right) \leq t f(y)+(1-t) f\left(x_{n}\right)-f\left(x_{n}\right)=t\left(f(y)-f\left(x_{n}\right)\right)$. From (3.6), it follows that

$$
\left\langle u_{n}, y-x_{n}\right\rangle+f(y)-f\left(x_{n}\right) \geq 0 .
$$

Since $y$ is arbitrary, $x_{n}$ is a solution of GMVI (1.1). The proof is complete. 
Theorem 3.7. Let $K$ be a nonempty and closed convex subset of $B$ and $f: K \rightarrow R \cup\{+\infty\}$ be a proper and convex lower semicontinuous function. Suppose that $F: K \rightarrow 2^{B^{*}}$ is $f$-quasimonotone, and for every $x \in K$, there exists a convex neighborhood $V_{x}$ of $x$ and a $f$-upper sign-continuous operator $S_{x}: V_{x} \cap K \rightarrow 2^{B^{*}}$ with nonempty, $w^{*}$-compact and convex values satisfying $S_{x}(y) \subseteq F(y) \forall y \in V_{x} \cap K$. If the condition $\left(C_{2}\right)$ holds, then GMVI (1.1) has a solution.

Proof. First, we will show that if there is no solution of GMVI (1.1), then it follows from the condition $\left(C_{2}\right)$ that the condition $\left(C_{1}\right)$ holds. Since the condition $\left(C_{2}\right)$ holds, then given any $\hat{x} \in B$, for any $\left\{x_{n}\right\} \subset K$ with $\left\|x_{n}\right\| \rightarrow+\infty$ as $n \rightarrow+\infty$, and any $\left\{u_{n}\right\}$ with $u_{n} \in F\left(x_{n}\right)$, there exist a positive integer $N$ and $\bar{x} \in K$ such that $\|\bar{x}-\hat{x}\|<\left\|x_{N}-\hat{x}\right\|$ and

$$
\left\langle u_{N}, \bar{x}-x_{N}\right\rangle+f(\bar{x})-f\left(x_{N}\right) \leq 0 .
$$

Since there is no solution of GMVI (1.1), $x_{N}$ is not a solution of GMVI (1.1), that is, there exists $y_{0} \in K$ such that

$$
\left\langle u_{N}, y_{0}-x_{N}\right\rangle+f\left(y_{0}\right)-f\left(x_{N}\right)<0 .
$$

Since $\left\|x_{N}-\hat{x}\right\|-\|\bar{x}-\hat{x}\|>0$, we can select sufficiently small $a_{0} \in(0,1)$ such that

$$
a_{0}\left\|y_{0}-\hat{x}\right\| \leq\left(1-a_{0}\right)\left(\left\|x_{N}-\hat{x}\right\|-\|\bar{x}-\hat{x}\|\right) .
$$

Put $z=\left(1-a_{0}\right) \bar{x}+a_{0} y_{0}$. Since $K$ is convex, we have $z \in K$, and

$$
\|z-\hat{x}\| \leq\left(1-a_{0}\right)\|\bar{x}-\hat{x}\|+a_{0}\left\|y_{0}-\hat{x}\right\| \leq\left(1-a_{0}\right)\left\|x_{N}-\hat{x}\right\| \leq\left\|x_{N}-\hat{x}\right\| .
$$

Since $f$ is convex, by (3.7) and (3.8) we get

$$
\begin{aligned}
\left\langle u_{N},\right. & \left.z-x_{N}\right\rangle+f(z)-f\left(x_{N}\right) \\
& \leq\left(1-a_{0}\right)\left\langle u_{N}, \bar{x}-x_{N}\right\rangle+a_{0}\left\langle u_{N}, y_{0}-x_{N}\right\rangle+\left(1-a_{0}\right) f(\bar{x})+a_{0} f\left(y_{0}\right)-f\left(x_{N}\right) \\
& =\left(1-a_{0}\right)\left\langle u_{N}, \bar{x}-x_{N}\right\rangle+\left(1-a_{0}\right)\left(f(\bar{x})-f\left(x_{N}\right)\right)+a_{0}\left\langle u_{N}, y_{0}-x_{N}\right\rangle+a_{0}\left(f\left(y_{0}\right)-f\left(x_{N}\right)\right) \\
& \leq a_{0}\left\langle u_{N}, y_{0}-x_{N}\right\rangle+a_{0}\left(f\left(y_{0}\right)-f\left(x_{N}\right)\right)<0 .
\end{aligned}
$$

Hence the condition $\left(C_{1}\right)$ holds. Theorem 3.6 implies that GMVI 1.1 has a solution. This is a contradiction. Therefore, GMVI (1.1) has a solution. The proof is complete.

Combining Remark 3.5 and Theorems 3.6 and 3.7, we obtain the following corollary.

Corollary 3.8. Let $K$ be a nonempty and closed convex subset of $B$ and $f: K \rightarrow R \cup\{+\infty\}$ be a proper and convex lower semicontinuous function. Suppose that $F: K \rightarrow 2^{B^{*}}$ is $f$-quasimonotone, and for every $x \in K$, there exists a convex neighborhood $V_{x}$ of $x$ and a $f$-upper sign-continuous operator $S_{x}: V_{x} \cap K \rightarrow 2^{B^{*}}$ with nonempty, $w^{*}$-compact and convex values satisfying $S_{x}(y) \subseteq F(y) \forall y \in V_{x} \cap K$. If one of the conditions $\left(C_{3}-C_{5}\right)$ holds, then GMVI (1.1) has a solution.

Remark 3.9. We would like to mention that the differences between Theorems 3.6 and 3.7 and Theorem 4.2 in [25] lie in the following aspects: Theorem 4.2 in [25] asked the condition (i) and (ii) involved the Lyapunov functional $G$ introduced in 23 hold, while Theorems 3.6 and 3.7 of this paper have no any assumption about the Lyapunov functional $G$; (ii) Theorem 4.2 in [25] required that $B$ is a reflexive and smooth Banach space, but Theorems 3.6 and 3.7 need $B$ is only a reflexive Banach space. Moreover, Theorems 3.6 and 3.7 improve and extend Theorem 4.1 in [20] and Theorem 4.1 in [21] in the following aspects: (i) $B$ may not be strictly convex and smooth; (ii) $F$ may not equal to $J-T$ and may not be a compact mapping. (iii) By Remark 3.5. the condition $\left(C_{4}\right)$ and $\left(C_{2}\right)$ is weaker than the one that $L_{<}(\hat{x})$ is bounded (possibly empty) in Theorem 4.1 of [20].

From Theorems 3.6, 3.7 and Remark 3.5, it is easy to obtain the following corollary. 
Corollary 3.10. Let $K$ be a nonempty bounded and closed convex subset of $B$. Suppose that $F: K \rightarrow 2^{B^{*}}$ is quasimonotone, and for every $x \in K$, there exists a convex neighborhood $V_{x}$ of $x$ and a upper sign-continuous operator $S_{x}: V_{x} \cap K \rightarrow 2^{B^{*}}$ with nonempty, $w^{*}$-compact and convex values satisfying $S_{x}(y) \subseteq F(y) \forall y \in$ $V_{x} \cap K$. Assume that one of the following conditions holds:

$\left(C_{1}^{\prime}\right)$ Given $\hat{x} \in B$ and for any $\left\{x_{n}\right\} \subset K$ with $\left\|x_{n}\right\| \rightarrow+\infty$ as $n \rightarrow+\infty$, and any $\left\{u_{n}\right\}$ with $u_{n} \in F\left(x_{n}\right)$, there exist a positive integer $N$ and $\bar{x} \in K$ such that $\|\bar{x}-\hat{x}\| \leq\left\|x_{N}-\hat{x}\right\|$ and

$$
\left\langle u_{N}, \bar{x}-x_{N}\right\rangle<0
$$

$\left(C_{2}^{\prime}\right)$ Given $\hat{x} \in B$ and for any $\left\{x_{n}\right\} \subset K$ with $\left\|x_{n}\right\| \rightarrow+\infty$ as $n \rightarrow+\infty$, and any $\left\{u_{n}\right\}$ with $u_{n} \in F\left(x_{n}\right)$, there exist a positive integer $N$ and $\bar{x} \in K$ such that $\|\bar{x}-\hat{x}\|<\left\|x_{N}-\hat{x}\right\|$ and

$$
\left\langle u_{N}, \bar{x}-x_{N}\right\rangle \leq 0
$$

$\left(C_{3}^{\prime}\right)$ Given $\hat{x} \in B$, there exists a constant $\rho>0$ such that, for any $x \in K$ with $\|x-\hat{x}\|>\rho$, there exists $\bar{x} \in K$ satisfying $\|\bar{x}-\hat{x}\| \leq\|x-\hat{x}\|$ and $\sup _{u \in F(x)}\langle u, \bar{x}-x\rangle<0 ;$

$\left(C_{4}^{\prime}\right)$ Given $\hat{x} \in B$, there exists a constant $\rho>0$ such that, for any $x \in K$ with $\|x-\hat{x}\|>\rho$, there exists $\bar{x} \in K$ satisfying $\|\bar{x}-\hat{x}\|<\|x-\hat{x}\|$ and $\sup _{u \in F(x)}\langle u, \bar{x}-x\rangle \leq 0 ;$

$\left(C_{5}^{\prime}\right)$ Suppose that there exists a vector $\tilde{x} \in K$ such that the set

$$
L_{<}(\tilde{x}):=\left\{x \in K: \inf _{u \in F(x)}\langle u, x-\tilde{x}\rangle<0\right\}
$$

is bounded (possibly empty).

Then GVI 1.3 has a solution.

Remark 3.11. Corollary 3.10 improves and extends Theorem 4.2 in 21] in the following aspects: (i) If $\hat{x}=0$, then the condition $\left(C_{4}^{\prime}\right)$ is precisely the condition $\left(C_{3}\right)$ in Theorem 4.2 of [21]. According to Remark 3.5 , we know the condition $\left(C_{2}^{\prime}\right)$ is weaker than the condition $\left(C_{3}\right)$ in Theorem 4.2 of [21]. (ii) $B$ may not be strictly convex and smooth; (iii) $F$ may not be equivalent to $J-T$ and may not be a compact mapping. Thus Corollary 3.10 also improves and extends Theorem 5 in [13] and Corollary 4.3 in [17].

Corollary 3.10 extends and improves Theorems 3.4 and 3.6 in [14] in the following aspects: (i) the Banach space $B$ may not has a Fréchet differentiable norm; (ii) $F(B)=\cup_{x \in K} F(x)$ may not be compact in $B^{*}$; (iii) the upper semicontinuity of the mappings is relaxed to the sign-upper continuity; (iv) the weakly convergent sequence in $K$ may not be norm convergent.

We note that the differences between Theorem 3.2 in [22] and Corollary 3.10 lie in the following aspects: (i) Theorem 3.2 in 22 asked $G\left(y_{0}\right)$ is compact and the set-valued mapping $T$ is upper semicontinuous, while Corollary 3.10 of this paper has no any compactness assumption and requires $F$ is a quasimonotone mapping; (ii) Theorem 3.2 in 22] required that $E$ is a reflexive and smooth Banach space, but Corollary 3.10 needs $B$ is only a reflexive Banach space.

We note that the differences between Theorem 2.1 in [3] and Corollary 3.10 lie in the following aspects: (i) although we assume that $B$ is a reflexive Banach space, we don't suppose that $K \cap \bar{B}\left(0, \rho^{\prime}\right)$ is nonempty weakly compact in Theorem 2.1 of [3]. In addition, if $\hat{x}=0$, then the condition $\left(C_{4}^{\prime}\right)$ reduces to the condition (4) in Theorem 2.1 of 3 .

\section{Acknowledgements}

This work was supported by Sichuan applied basic research projects (2010JY0067), the key program of the fundamental research funds for the central universities (SWJTU09ZT36), the fundamental research funds for the central universities(2682015CX061), the NSF of China (Tianyuan funds for Mathematics, NO.11526170) 


\section{References}

[1] K. Addi, S. Adly, D. Goeleven, H. Saoud, A sensitivity analysis of a class of semi-coercive variational inequalities using recession tools, J. Global Optim., 40 (2008), 7-27. 1

[2] Y. I. Alber, Metric and generalized projection operators in Banach spaces: Properties and applications, in: A. Kartsatos (Ed.), Theory and Applications of Nonlinear Operators of Accretive and Monotone Type, Marcel Dekker, New York, (1996). 1, 1

[3] D. Aussel, N. Hadjisavvas, On Quasimonotone variational inequalities, J. Optim. Theory Appl., 121 (2004), 445-450. 3, 3.4, 3.5, 3.11

[4] C. Baiocchi, A. Capelo, Variational and quasivariational inequalities: applications to free-Boundary problems, John Wiley and Sons, New York, (1984). 1, 1 .

[5] E. Blum, W. Oettli, From optimization and variational inequalities to equilibrium problems, Math. Student, 63 (1994), 123-145. 1

[6] F. E. Browder, Nonlinear maximal monotone mappings in Banach spaces, Math. Ann., 175 (1968), 89-113. 1. 1 ,

[7] F. Facchinei, J. S. Pang, Finite dimensional variational inequalities and complementarity problems, SpringerVerlag, New York, (2003). 1 .

[8] K. Fan, A generalization of Tychonoff's fixed point theorem, Math. Ann., 142 (1961), 305-310. 2.6

[9] N. Hadjisavvas, Continuity and Maximality Properties of Pseudomonotone Operators, J. Convex Anal., 10 (2003), 465-475. 1, 2.3

[10] N. Hadjisavvas, S. Schaible, N. C. Wong, Pseudomonotone operators: a survey of the theory and its applications, J. Optim. Theory Appl., 152 (2012), 1-20. 1

[11] J. Y. Han, Z. H. Huang, S. C. Fang, Solvability of variational inequality problems, J. Optim. Theory Appl., 122 (2004), 501-520. 1. 3

[12] G. Isac, Complementarity problems and variational inequalities: A unified approach of solvability by and implicit Leray-Schauder-type alternative, J. Glob. Optim., 31 (2005), 405-420. 3.5

[13] G. Isac, J. Li, Exceptional family of elements and the solvability of complementarity problems in uniformly smooth and uniformly convex Banach spaces, J. Zhejiang Univ. Sci., 6 (2005), 289-295. 1] 3, 3.11]

[14] P. Junlouchai, S. Plubtieng, Existence of solutions for generalized variational inequality problems in Banach spaces, Nonlinear Anal., 74 (2011), 999-1004. 1, 1, 3.4, 3, 3.11

[15] I. V. Konnov, E. O. Volotskaya, Mixed variational inequalities and economic equilibrium problems, J. Appl. Math., 2 (2002), 289-314. 1

[16] J. Li, N. J. Huang, Exceptional families of elements for set-valued mappings: An application to nonlinear complementarity problems, Appl. Math. Lett., 21 (2008), 42-46. 11, 3

[17] J. Li, J. Whitaker, Exceptional family of elements and solvability of variational inequalities for mappings defined only on closed convex cones in Banach spaces, J. Math. Anal. Appl., 310 (2005), 254-261. 1, 3 , 3.11

[18] M. Sion, On general minimax theorems, Pacific J. Math., 8 (1958), 171-176. 2.7

[19] G. J. Tang, N. J. Huang, Existence theorems of the variational-hemivariational inequalities, J. Global Optim., 56 (2013), 605-622. 1, 2.5, 3

[20] Z. B. Wang, N. J. Huang, Degree theory for a generalized set-valued variational inequality with an application in Banach spaces, J. Global Optim., 49 (2011), 343-357. 1, $1,3.9$

[21] Z. B. Wang, N. J. Huang, Exceptional family of elements for a generalized set-valued variational inequality in Banach spaces, Optimization, 63 (2014), 167-180. 1, 3, 3.9, 3.11

[22] M. M. Wong, Q. H. Ansari, J. C. Yao, Existence of solutions of generalized variational inequalities in reflexive Banach spaces, Appl. Math. Lett., 22 (2009), 197-201. 1. 3. 3.11

[23] K. Q. Wu, N. J. Huang, The generalized $f$-projection operator with an application, Bull. Austral. Math. Soc., 73 (2006), 307-317. 1, $1,3.9$

[24] K. Q. Wu, N. J. Huang, Properties of the generalized $f$-projection operator and its applications in Banach spaces, Comput. Math. Appl., 54 (2007), 399-406. 1, 1

[25] K. Q. Wu, N. J. Huang, The generalized $f$-projection operator and set-valued variational inequalities in Banach spaces, Nonlinear Anal., 71 (2009), 2481-2490. 1, 1, 3.9

[26] J. Yu, H. Yang, Existence of solutions for generalized variational inequality problems, Nonlinear Anal., 71 (2009), e2327-e2330. 3.5

[27] L. C. Zeng, J. C. Yao, Existence theorems for variational inequalities in Banach spaces, J. Optim. Theory Appl., $132(2007), 321-337.1$

[28] R. Y. Zhong, N. J. Huang, Stability analysis for minty mixed variational inequality in reflexive Banach spaces, J. Optim. Theory Appl., 147 (2010), 454-472. 1, 1, 2.5

[29] R. Y. Zhong, N. J. Huang, Strict feasibility for generalized mixed variational inequality in reflexive Banach spaces, J. Optim. Theory Appl., 152 (2012), 696-709. 1, 1, 2.5, 3 